\title{
An Improved Nonlinear Aerodynamic Derivative Model of Aircraft at High Angles of Attack
}

\author{
Mi Baigang $(\mathbb{D}$ and Yu Jingyi \\ School of Aeronautics, Northwestern Polytechnical University, Xi'an, Shaanxi 710072, China \\ Correspondence should be addressed to Mi Baigang; mibaigang@163.com
}

Received 26 July 2021; Accepted 27 August 2021; Published 10 September 2021

Academic Editor: Jinyang $\mathrm{Xu}$

Copyright (C) $2021 \mathrm{Mi}$ Baigang and Yu Jingyi. This is an open access article distributed under the Creative Commons Attribution License, which permits unrestricted use, distribution, and reproduction in any medium, provided the original work is properly cited.

\begin{abstract}
The classical aerodynamic derivative model is widely used in flight dynamics, but its application is extremely limited in cases with complicated nonlinear flows, especially at high angles of attack. A modified nonlinear aerodynamic derivative model for predicting unsteady aerodynamic forces and moments at a high angle of attack is developed in this study. We first extend the higher-order terms to describe the nonlinear characteristics and then introduce three more influence parameters, the initial angle of attack, the reduced frequency, and the oscillation amplitude, to correct the constant aerodynamic derivative terms that have higher-order polynomials for these values. The improved nonlinear aerodynamic derivative model was validated by using the NACA 0015 airfoil and the F-18 model. The results show that the improved model has a higher prediction ability at high angles of attack and has the ability to predict the aerodynamic characteristics of other unknown states based on known unsteady aerodynamic data, such as the initial angle of attack, reduced frequency, and oscillation amplitude.
\end{abstract}

\section{Introduction}

The unsteady aerodynamic characteristic requirements at high angles of attack for the next generation aircraft are increasing quickly, and the complicated unsteady aerodynamics caused by dynamic motion at high angles of attack has become one of the current research hotspots $[1,2]$. At this time, the unsteady aerodynamic force is highly nonlinear, and the whole dynamic flow field is accompanied by complex vortex motion, evolution, and mutual interference, thus forming a complex nonlinear system $[3,4]$. To obtain the detailed changes in the aerodynamic forces within this dynamic process, large-scale CFD simulations and wind tunnel tests should be utilized [5, 6]. However, the large costs of both CFD and experiments seriously restrict the process of modern aircraft design. Therefore, more attention has been given on how to obtain acceptable aerodynamic data through limited calculations or tests and establish a relatively accurate mathematical model to predict the aerodynamic characteristics of the dynamic motion at high angles of attack, that is, unsteady aerodynamic modeling $[7,8]$. The commonly used unsteady aerodynamic modeling methods can be divided into two categories. One is the traditional method based on the characteristics of the flow field, such as aerodynamic derivative models $[9,10]$, state space models $[1,11]$, differential equation models $[12,13]$, and integrated models [14-17]. These models are developed based on the physical mechanisms of the flow field around the aircraft, and different models have different characterization abilities. The integrated model is built by dividing the dynamic motion into infinite step motions, and it is a mathematically complete model. However, this model is difficult to identify. The other models can be considered special forms of the integrated model and show strong prediction and modeling ability in aerodynamic performance evaluation. The other method is based on modern models that use intelligent algorithms for prediction, such as fuzzy logic models [18-21]. The traditional method is simple and can characterize the flow field characteristics more or less, but parameter identification is difficult and the universality is poor. Intelligent algorithms ignore the specific relationship between the input and output, and they use AI algorithms to build implicit relations. It seems that AI models are of little help in understanding flow field characteristics. However, 
they are still a focus due to their good applicability to both 2-D and 3-D configurations and subsonic and supersonic cases. Currently, both conventional and modern intelligent methods are undergoing constant development and improvement.

Among the conventional methods, the most widely used is the aerodynamic derivative model. As early as 1911, this model emerged alongside the advent of aviation technology. In this model, it is assumed that the aerodynamic forces can be expressed as a function of a series of variables of the aircraft. Thus, by using a Taylor series expansion, an aerodynamic derivative model is built and developed. As the cross terms among the longitudinal and lateral forces and moments in the model are ignored, the flight dynamics can be decoupled and simplified. Moreover, the static and dynamic stability criteria in classical flight dynamics are directly determined by the various aerodynamic derivatives [9]. It can be considered that the aerodynamic derivative model determines the basis of traditional flight mechanics research.

The aerodynamic derivative model is based on a linear system, so the aerodynamic expression of unsteady dynamic motion at low angles of attack is more accurate, and it is not suitable for nonlinear situations with high angles of attack [10]. However, this model is still worth attention because of its simple form and easy coupling with flight quality analysis.

This paper studies the traditional aerodynamic derivative model, deeply analyzes the shortcomings of the original model in the prediction of dynamic aerodynamic characteristics at high angles of attack, improves its expression ability at high angles of attack, and expands its angles of attack application range. Finally, the improved model is verified and analyzed by using the dynamic motion of the NACA 0015 airfoil in different cases.

\section{Classical Aerodynamic Derivative Model}

Taking the longitudinal motion as an example, the classical aerodynamic derivative model [9] can be described by

$$
C_{m}=C_{m 0}+C_{m \alpha} \cdot \Delta \alpha+C_{m \dot{\alpha}} \cdot \Delta \dot{\alpha}+C_{m q} \cdot q+\Delta
$$

The above formula ignores the influence of the rudder surface and only expands the pitch moment coefficient as a function of the angle of attack and pitching angular velocity, where $C_{m a}$ and $C_{m \dot{a}}$ are the static derivative and acceleration derivative, respectively, and $C_{m q}$ denotes the damping derivative.

Equation (1) is usually written in a dimensionless form:

$$
C_{m}=C_{m 0}+C_{m \alpha} \cdot \Delta \alpha+C_{m \dot{\alpha}} \cdot \frac{\Delta \dot{\alpha} c}{2 V}+C_{m q} \cdot \frac{q c}{2 V}+\Delta .
$$

When the velocity of the free stream remains unchanged, the rate change of the angle of attack is consistent with the expression of the pitch angular velocity; therefore,

$$
C_{m}=C_{m 0}+C_{m \alpha} \cdot \Delta \alpha+\left(C_{m \dot{\alpha}}+C_{m q}\right) \cdot \frac{q c}{2 V}+\Delta .
$$

Omitting the higher-order term, the commonly used aerodynamic derivative model can be obtained:

$$
\begin{aligned}
C_{m} & =C_{m 0}+C_{m \alpha} \cdot \Delta \alpha+\bar{C}_{m q} \cdot \frac{q c}{2 V}, \\
\bar{C}_{m q} & =C_{m \dot{\alpha}}+C_{m q} \cdot
\end{aligned}
$$

The aerodynamic derivative model takes the static and dynamic derivatives as constant values, so the aerodynamic force caused by a given motion can be predicted only by knowing the motion form and the initial aerodynamic force value. The premise of the model is small disturbances, and the flow field characteristics are basically linear. If the maneuvering flight of the aircraft produces some strong time-dependent flows at high angles of attack, the model is no longer applicable.

\section{Classical Aerodynamic Derivative Model Validation}

3.1. Simulation Model of the NACA 0015 Airfoil. Taking the two-dimensional NACA 0015 airfoil with medium thickness as the object, the unsteady pitch oscillation at low and high angles of attack is numerically simulated to investigate the aerodynamic prediction ability of the original aerodynamic derivative model. The overview of the mesh is shown in Figure 1. The first layer (boundary layer) grid keeps the parameter $y+\approx 1.1$ to satisfy the requirement of viscous effect simulation. The Mach number is $0.2(H=0)$. Rigid dynamic mesh technology is used to describe the unsteady motion of the airfoil. As all mesh nodes move with the same form, the mesh will not be deformed, and the computation can be more accurate and effective. The statically calculated lift coefficient is shown in Figure 2. When the angle of attack of the airfoil is approximately $17.5^{\circ}$, the lift coefficient reaches the maximum. At this time, the corresponding flow field shows that a small separation zone is generated at the trailing edge. As the angle of attack increases, the separation zone expands and moves toward the leading edge of the airfoil. Then, the airfoil begins to stall. Please note that the NACA 0015 airfoil is a relatively thick airfoil; therefore, the stall begins from the trailing edge. For other airfoils, the flow separation can be different. However, these factors have no influence on the aerodynamic models.

3.2. Unsteady Aerodynamic Prediction at Small Angles of Attack. The aerodynamic derivative model is used to predict the unsteady aerodynamic force at small angles of attack. First, we need to find each derivative in the model. In this paper, the derivative model with an initial angle of attack of $0^{\circ}$ is used to predict the aerodynamic force at an initial angle of attack of $4^{\circ}$. This is done because we determine that the flow characteristics between the two cases are nearly the same and the aerodynamic parameters can also be considered the same with each other, and then the two cases can be presented with the same aerodynamic derivative model. The movement mode of forced oscillation is 


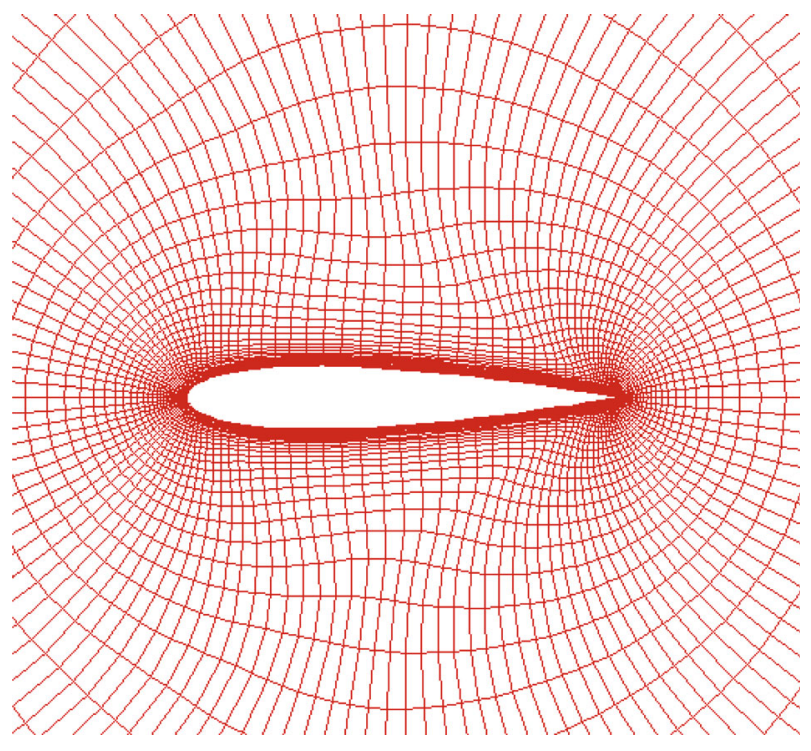

FIgURE 1: The computed mesh.

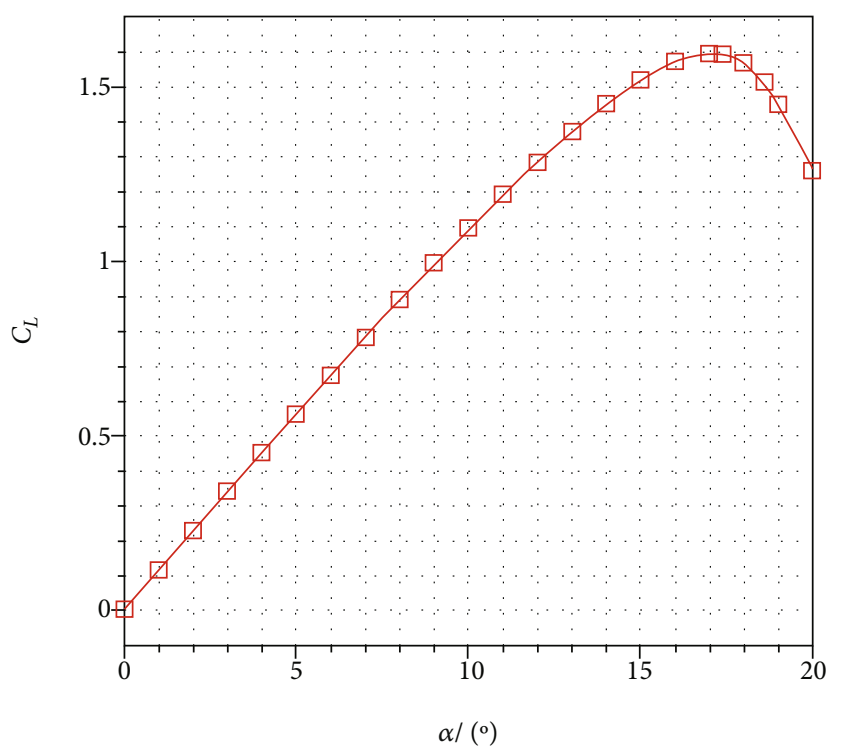

FIgURE 2: The statically calculated lift coefficient.

$$
\alpha=\alpha_{0}+\Delta \alpha=\alpha_{0}+\alpha_{m} \sin (\omega t)
$$

where $\alpha_{m}$ is the oscillation amplitude and the reduced frequency of $k$ is defined as $\omega c / 2 V$. The oscillation amplitude and frequency values are 1 and 0.08 , respectively.

The comparisons of the lift and pitch moment coefficients predicted by the aerodynamic derivative model and CFD calculation are shown in Figure 3. The comparison showed that there is good agreement between the predictions and the CFD calculations at low angles of attack, which indicates that the aerodynamic derivative model can accurately predict the unsteady aerodynamic force of a given motion at a low angle of attack and small amplitude oscillation. Note also that the values of the static and dynamic derivatives at $\alpha=0^{\circ}$ can still be used at $\alpha=4^{\circ}$, which further shows that the aerodynamic derivatives have little change in the linear range of low angles of attack. This is also an important reason for the convenient application of the aerodynamic derivative model.

3.3. Unsteady Aerodynamic Prediction at High Angles of Attack. When the initial angle of attack is increased to $\alpha=$ $20^{\circ}$, the airfoil under static conditions has entered the stall state, and the small amplitude pitching oscillation is still carried out at this angle of attack. The lift and pitch moment coefficients predicted by using the aerodynamic derivative model are shown in Figure 4. According to the calculated values, the hysteresis surrounding the direction changes from counterclockwise to clockwise, indicating that the flow field has become divergent. However, the prediction result of the aerodynamic derivative model is still counterclockwise, which shows that the model is no longer applicable.

It is concluded that for unsteady motion whose initial angle of attack is below the medium, the flow field in the whole process is basically linear or weakly nonlinear, and the nature of the flow field does not change. Therefore, the aerodynamic derivative could accurately predict the aerodynamic changes. However, when the initial angle of attack increases to the point where the flow field has strong separation or stall characteristics, the flow field is strongly nonlinear, and the applicability of the aerodynamic derivative model becomes poor or even cannot be used.

\section{Improved Nonlinear Aerodynamic Derivative Model}

4.1. Nonlinear Correction 1: Expanding Higher-Order Terms. In the original aerodynamic derivative model, the high-order derivative terms, such as $C_{m \dot{q}}, C_{m \ddot{\alpha}}, C_{m \dot{q}}, \cdots$, are ignored. According to the Taylor expansion principle, the higher the order of expansion, the closer it can approach the original function. Therefore, a new nonlinear model is established considering these high-order terms [10]. Since the incoming flow velocity is constant, there is a relationship between the pitch angular velocity and the change rate of the angle of attack:

$$
\begin{gathered}
\dot{\alpha}=q, \\
\ddot{\alpha}=\dot{q}, \\
\ddot{\alpha}=\ddot{q}, \\
\ldots
\end{gathered}
$$

The aerodynamic derivatives $C_{m 0}, C_{m \alpha}, C_{m \dot{\alpha}}, \cdots, C_{m q}$, $C_{m \dot{q}}, \cdots$ in the model are expressed by $C_{1}, C_{2}, C_{3}, \cdots$, so the original aerodynamic derivative model can be written as

$$
C_{i}=C_{1}+C_{2} \alpha+C_{3} \dot{\alpha}+\Delta
$$

The second-, third-, and fourth-order higher-order terms are considered 


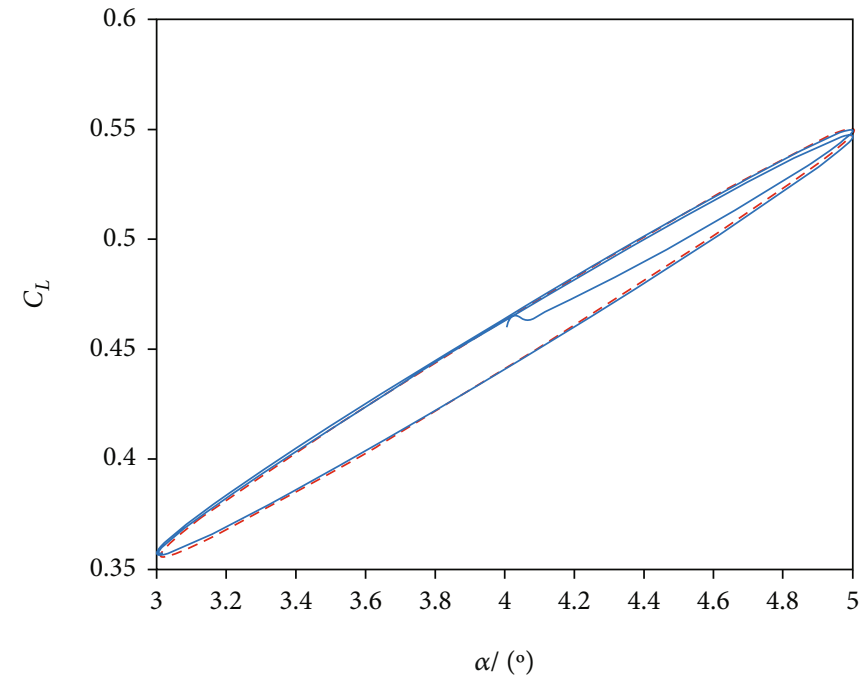

(a) Lift coefficient

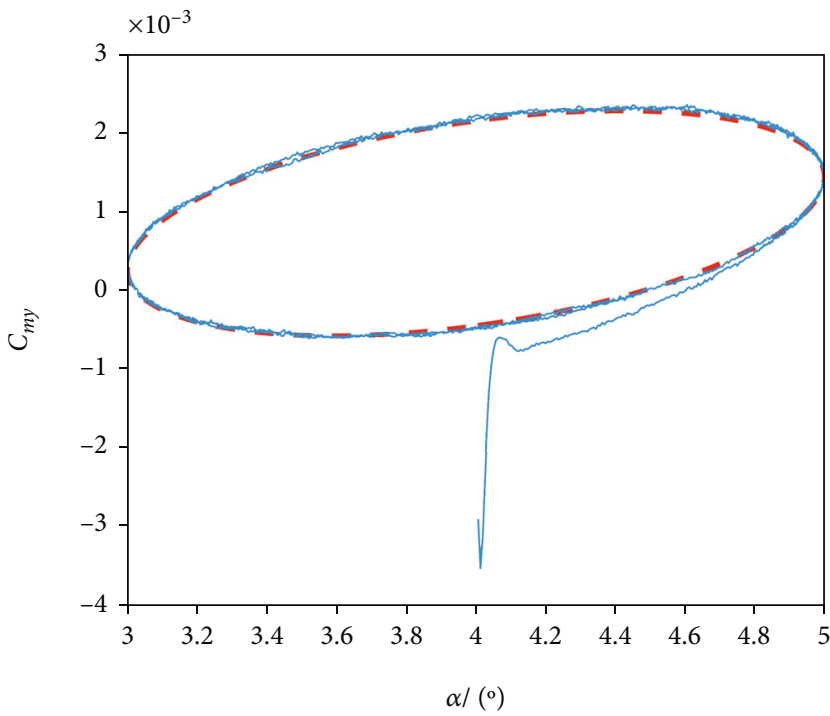

- - - Model data

— CFD result

(b) Pitch moment coefficient

FIGURE 3: Comparison between the calculated value of unsteady oscillation aerodynamic force and the predicted value of the model at the initial angle of attack of $\alpha=4^{\circ}$.

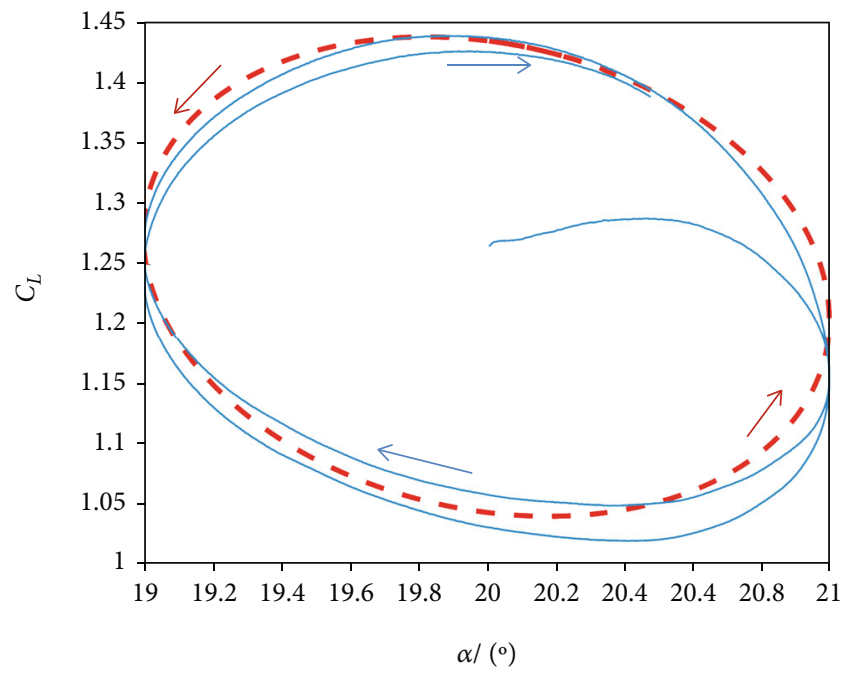

(a) Lift coefficient

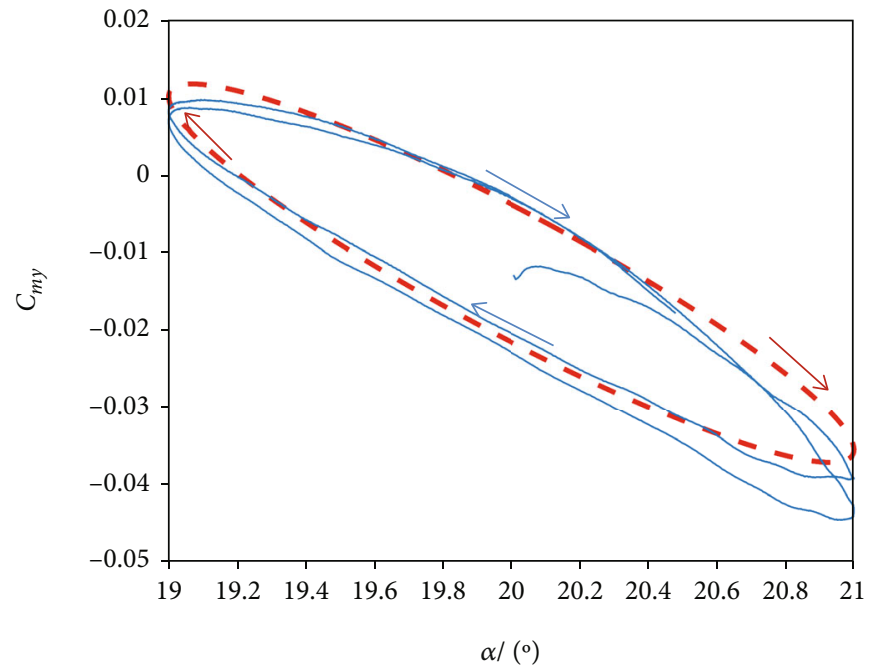

- - - Model data

- CFD result

(b) Pitch moment coefficient

FIGURE 4: Comparison between the calculated value of unsteady oscillation aerodynamic force and the predicted value of the model at the initial angle of attack of $20^{\circ}$. 

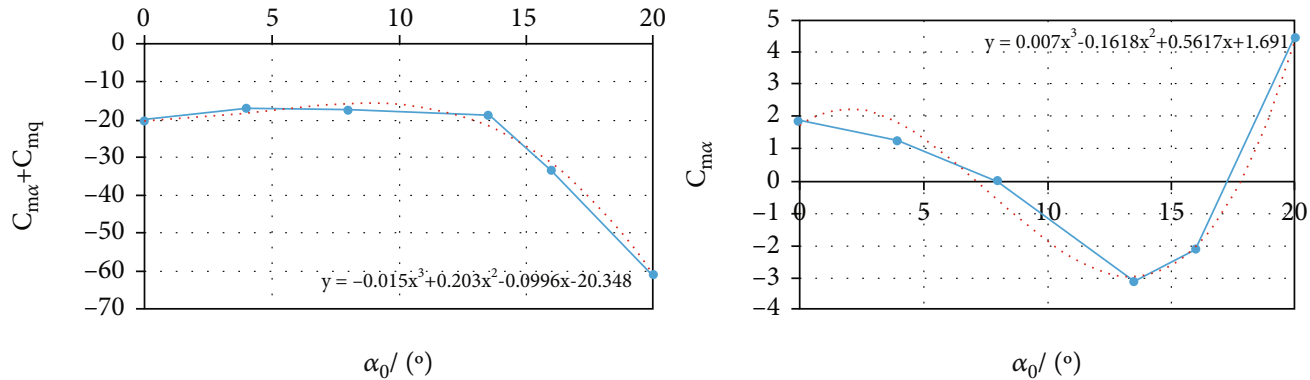

(a) Different initial angles of attack

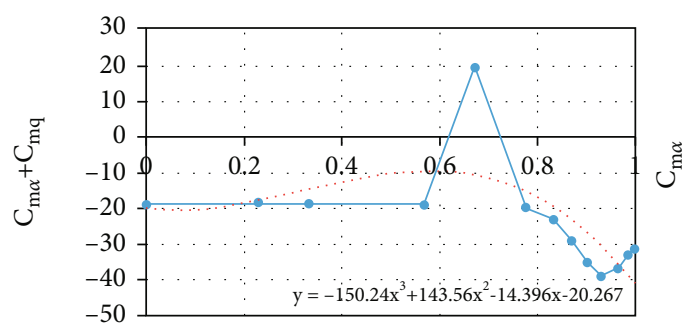

$k$

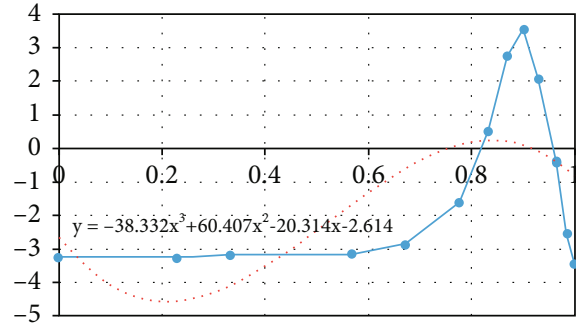

$k$

(b) Different reduced frequencies

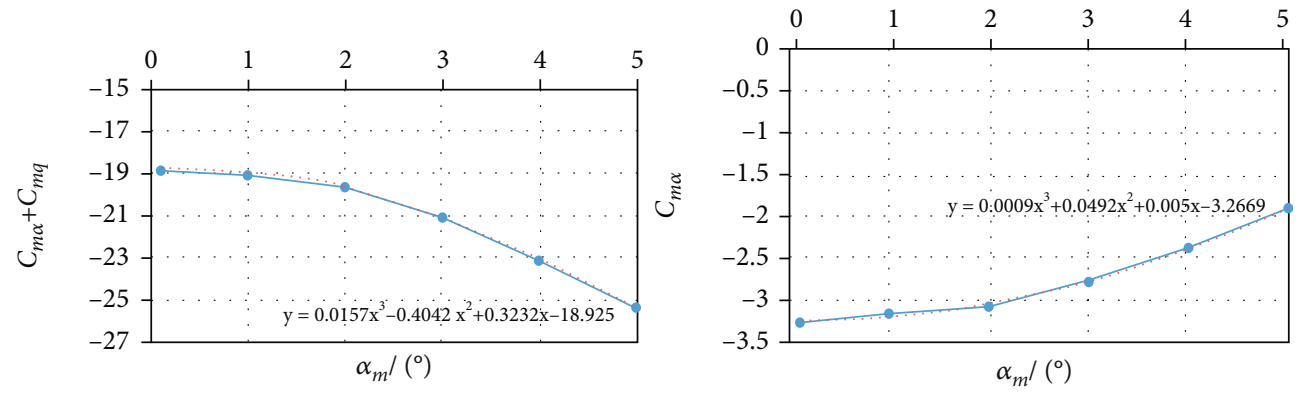

(c) Different pitching amplitudes

FIgURE 5: Static and dynamic and derivatives variation with different parameters of the HBS model.

$$
\begin{aligned}
C_{i}= & C_{0}+C_{1} \alpha+C_{2} \alpha^{2}+C_{3} \dot{\alpha}+C_{4}|\dot{\alpha}|+C_{5} \alpha \dot{\alpha}+C_{6} \alpha|\dot{\alpha}| \\
& +C_{7}|\dot{\alpha}| \alpha+C_{8} \dot{\alpha}^{2}, \\
C_{i}= & C_{0}+C_{1} \alpha+C_{2} \alpha^{2}+C_{3} \dot{\alpha}+C_{4}|\dot{\alpha}|+C_{5} \alpha \dot{\alpha}+C_{6} \alpha|\dot{\alpha}| \\
& +C_{7}|\dot{\alpha}| \dot{\alpha}+C_{8} \dot{\alpha}^{2}+C_{9} \alpha^{3}+C_{10} \dot{\alpha} \alpha^{2}+C_{11}|\dot{\alpha}| \alpha^{2} \\
& +C_{12} \dot{\alpha}^{2} \alpha+C_{13}|\dot{\alpha}| \dot{\alpha} \alpha+C_{14}|\dot{\alpha}| \dot{\alpha}^{2}+C_{15} \dot{\alpha}^{3}, \\
C_{i}= & C_{0}+C_{1} \alpha+C_{2} \alpha^{2}+C_{3} \dot{\alpha}+C_{4}|\dot{\alpha}|+C_{5} \alpha \dot{\alpha}+C_{6} \alpha|\dot{\alpha}| \\
& +C_{7}|\dot{\alpha}| \dot{\alpha}+C_{8} \dot{\alpha}^{2}+C_{9} \alpha^{3}+C_{10} \dot{\alpha} \alpha^{2}+C_{11}|\dot{\alpha}| \alpha^{2} \\
& +C_{12} \dot{\alpha}^{2} \alpha+C_{13}|\dot{\alpha}| \dot{\alpha} \alpha+C_{14}|\dot{\alpha}| \dot{\alpha}^{2}+C_{15} \dot{\alpha}^{3}+C_{16} \alpha^{4} \\
& +C_{17} \dot{\alpha} \alpha^{3}+C_{18}|\dot{\alpha}| \alpha^{3}+C_{19}|\dot{\alpha}| \dot{\alpha} \alpha^{2}+C_{20} \dot{\alpha}^{2} \alpha^{2} \\
& +C_{21} \dot{\alpha}^{3} \alpha+C_{22}|\dot{\alpha}|^{3} \alpha+C_{23}|\dot{\alpha}| \dot{\alpha}^{3}+C_{24} \dot{\alpha}^{4} .
\end{aligned}
$$

\subsection{Nonlinear Correction 2: Nonlinear Correction of} Aerodynamic Derivatives by Adding More Motion Parameters. According to the forced oscillation wind tunnel test, the aerodynamic derivative is closely related to the oscillation frequency, amplitude, and initial angle of attack at high angles of attack. Figure 5 shows the change in the longitudinal aerodynamic derivative of a canard layout aircraft with initial angle of attack, reduced frequency, and oscillation amplitude [22]. The value of the aerodynamic derivative is no longer constant in a certain range, with changes in the reduced frequency, oscillation amplitude, and initial angle of attack at high angles of attack. This is essentially different from the traditional aerodynamic derivative model, which represents the change in damping characteristics caused by the change in flow field properties.

Figure 5(a) shows that within a certain angle of attack, the aerodynamic derivative can be considered a constant value, but once the angle of attack exceeds the critical range, the dynamic derivative value clearly fluctuates, which indicates that the aerodynamic derivative term should be a function of the initial angle of attack. The reduced frequency represents the speed of the unsteady motion to some extent (Figure 5(b)). From the angle of high angles of attack, it also determines the hysteresis between the change in a vortex system structure and unsteady motion. If it is reflected in the large initial angle of attack, this effect is more clear. Figure 5(c) shows the influence of the oscillation amplitude on static and dynamic derivatives. The amplitude determines 


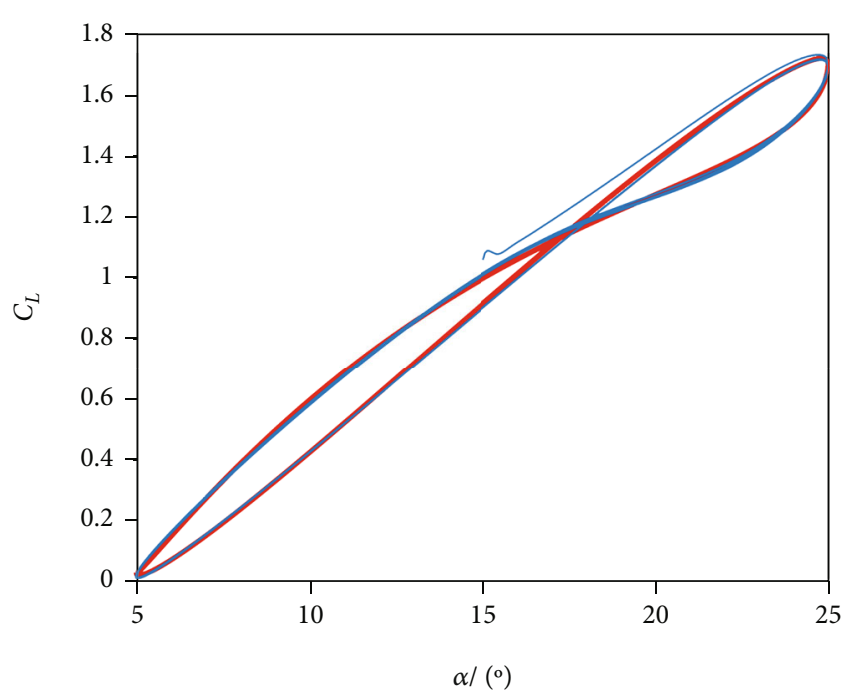

(a) Lift coefficient

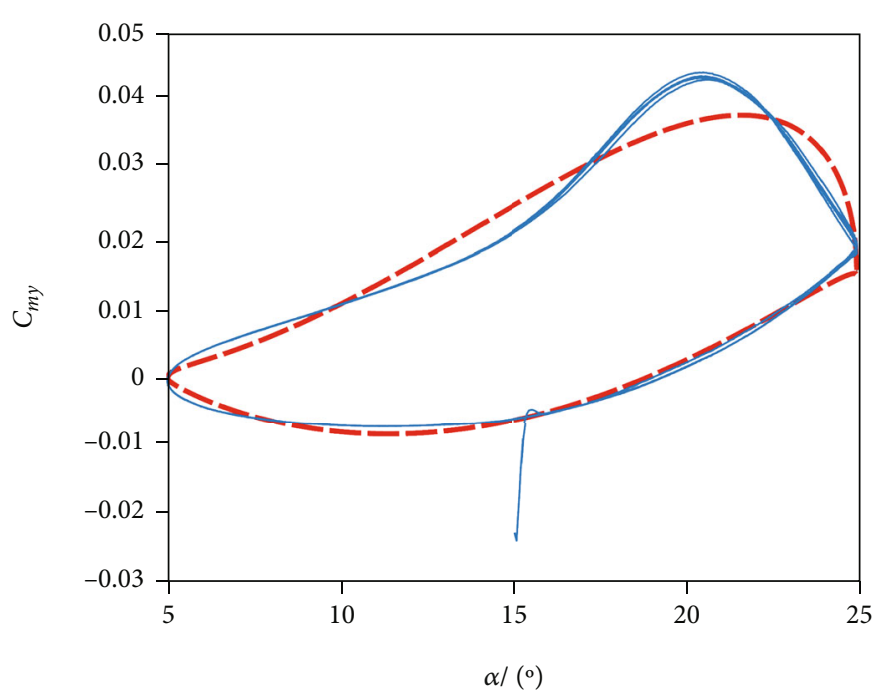

- - - Model data

C CFD result

(b) Pitch moment coefficient

Figure 6: Predictions of the second-order model $(k=0.06)$.

the range of the maximum and minimum angles of attack of unsteady motion and determines the flow pattern of the aircraft in unsteady motion. In the unsteady motion with high angles of attack and large amplitude, the more flow patterns that are crossed, the stronger the nonlinear characteristics.

Based on the expansion of the higher-order term in the previous section, the aerodynamic coefficient of the model is expressed as a function of the initial angle of attack $\alpha_{0}$, the reduced frequency $k$, and the oscillation amplitude $\alpha_{m}$. Using the existing data for analysis, the functional relationship between the angle of attack, reduced frequency, or oscillation amplitude and the static and dynamic derivatives can be described by cubic polynomials. In addition, some unsteady motions that are not harmonic vibrations can also be expressed as the relationship between the equivalent amplitude, reduced frequency, and aerodynamic derivative. There are two main reasons for choosing cubic polynomials. On the one hand, they have good universality; on the other hand, they are stable and have good fitting accuracy.

Referring to the above analysis, the functional relationship between the aerodynamic derivative terms and the angle of attack, reduced frequency, and oscillation amplitude should be written as

$$
\begin{aligned}
C_{i}= & a_{i 0}+a_{i 1} k+a_{i 2} k^{2}+a_{i 3} k^{3}+b_{i 0}+b_{i 1} \alpha_{m}+b_{i 2} \alpha_{m}{ }^{2} \\
& +b_{i 3} \alpha_{m}{ }^{3}+c_{i 0}+c_{i 1} \alpha+c_{i 2} \alpha^{2}+c_{i 3} c^{3} .
\end{aligned}
$$

Clearly, too many undetermined parameters are introduced, which increases the cost of subsequent modeling process identification, so it needs to be simplified. Considering the relationship between reduced frequency, oscillation amplitude, and angle of attack,

$$
\begin{aligned}
& k=\frac{\omega c}{2 V}, \\
& \dot{\alpha}=\alpha_{m} \omega \cos (\omega t), \\
& \dot{\alpha}=\frac{\alpha_{m} \omega \cos (\omega t) c}{2 V}=k \alpha_{m} \cos (\omega t) .
\end{aligned}
$$

Therefore, the reduced frequency and oscillation amplitude can be combined as one, and the simplified functional relationship can be written as

$$
C_{i}\left(\alpha_{0}, k, \alpha_{m}\right)=a_{i 0}+a_{i 1}\left(k \alpha_{m}\right)^{3}+a_{i 2}\left(k \alpha_{m}\right)^{2} \alpha_{0}+a_{i 3}\left(k \alpha_{m}\right) \alpha_{0}^{2}+a_{i 4} \alpha_{0}^{3} .
$$

Then, the corresponding second-, third-, and fourthorder models can be improved to

(1) The second-order model:

$$
\begin{aligned}
C_{i}= & C_{0}+C_{1}\left(\alpha_{0}, k, \alpha_{m}\right) \alpha+C_{2}\left(\alpha_{0}, k, \alpha_{m}\right) \alpha^{2}+C_{3}\left(\alpha_{0}, k, \alpha_{m}\right) \dot{\alpha} \\
& +C_{4}\left(\alpha_{0}, k, \alpha_{m}\right)|\dot{\alpha}|+C_{5}\left(\alpha_{0}, k, \alpha_{m}\right) \alpha \dot{\alpha}+C_{6}\left(\alpha_{0}, k, \alpha_{m}\right) \alpha|\dot{\alpha}| \\
& +C_{7}\left(\alpha_{0}, k, \alpha_{m}\right)|\dot{\alpha}| \alpha+C_{8}\left(\alpha_{0}, k, \alpha_{m}\right) \dot{\alpha}^{2} .
\end{aligned}
$$

(2) The third-order model: 


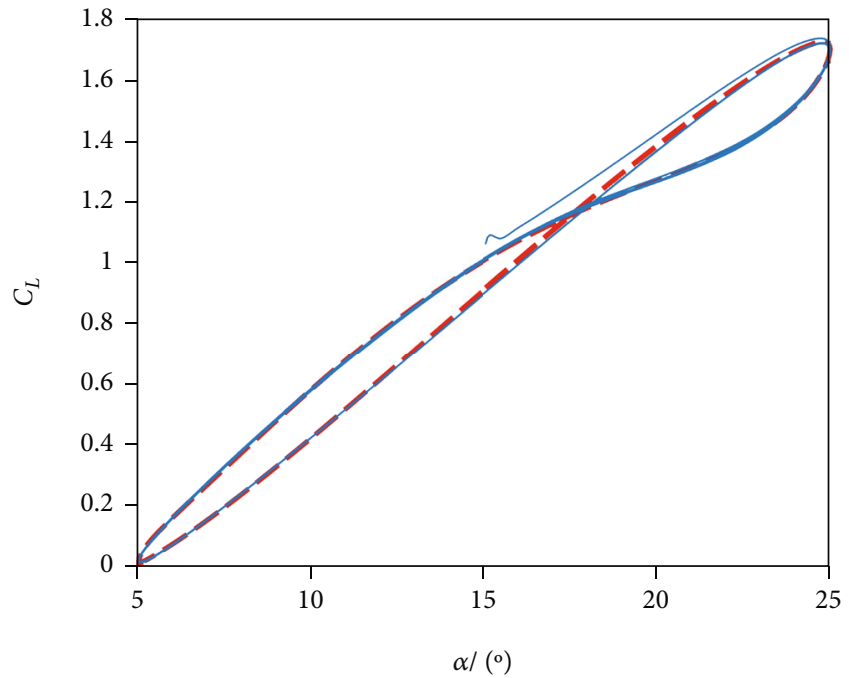

(a) Lift coefficient

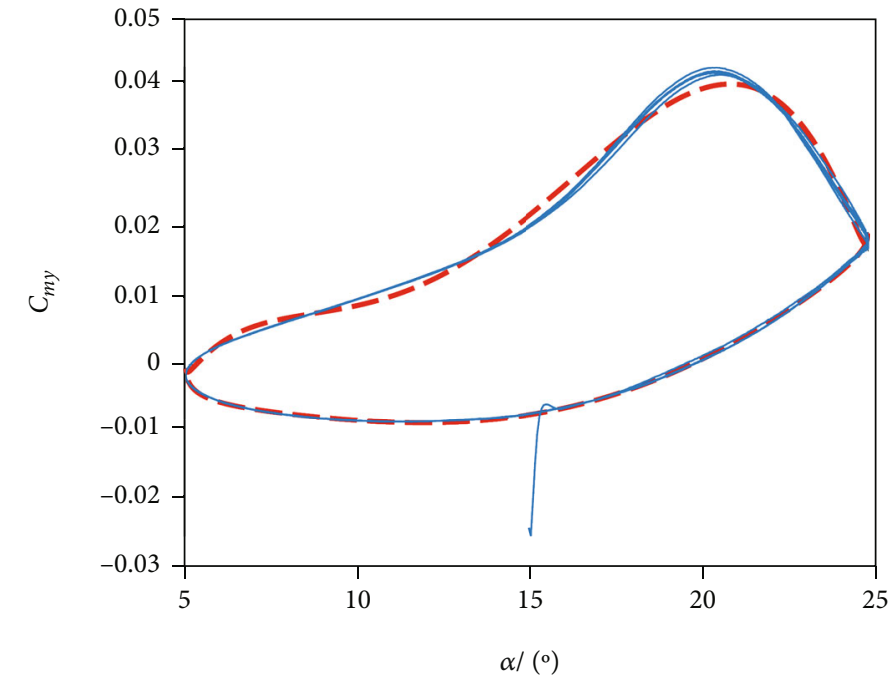

- - Model data

C CFD result

(b) Pitch moment coefficient

Figure 7: Predictions of the third-order model $(k=0.06)$.

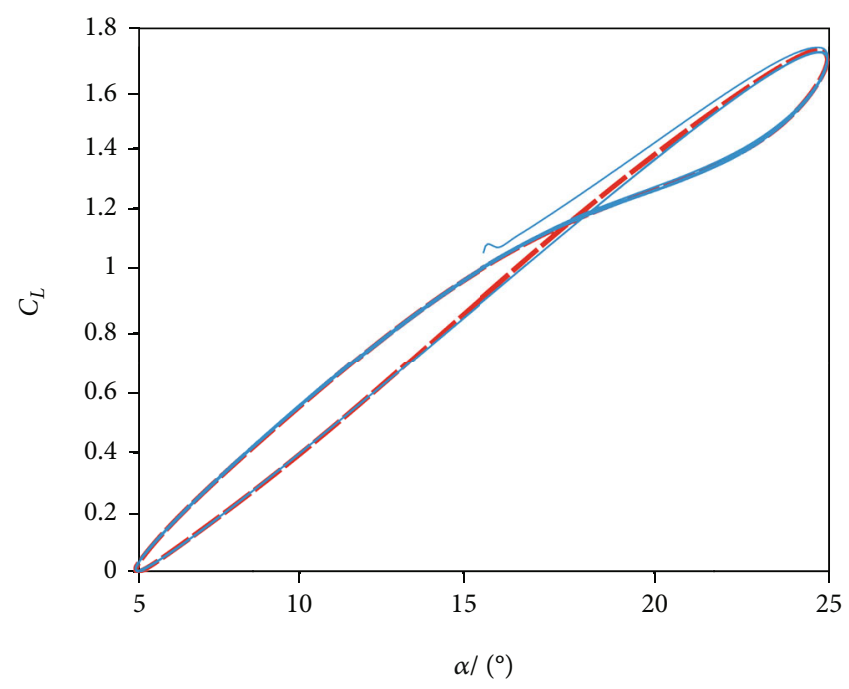

(a) Lift coefficient

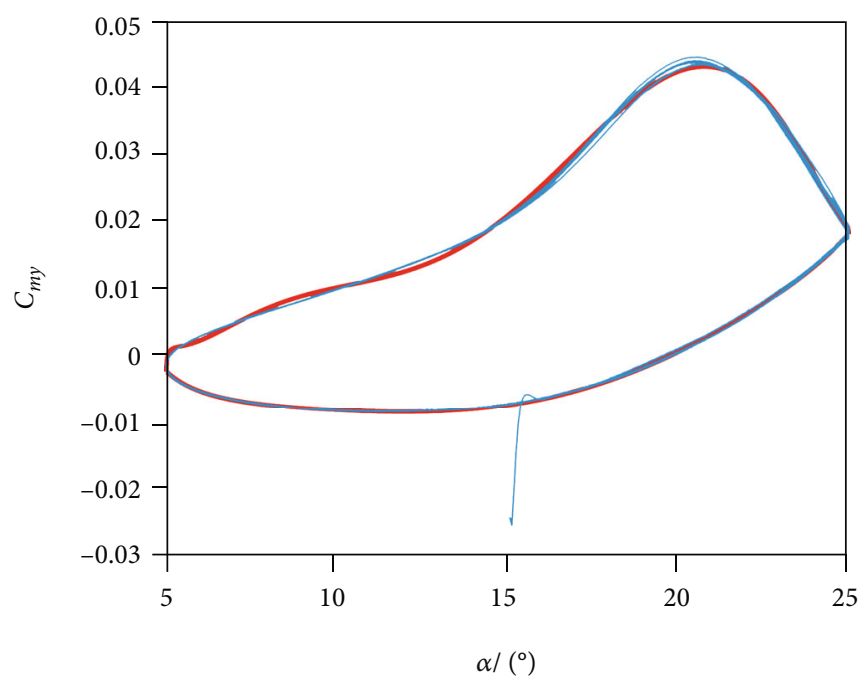

- - - Model data

- CFD result

Figure 8: Predictions of the fourth-order model $(k=0.06)$. 


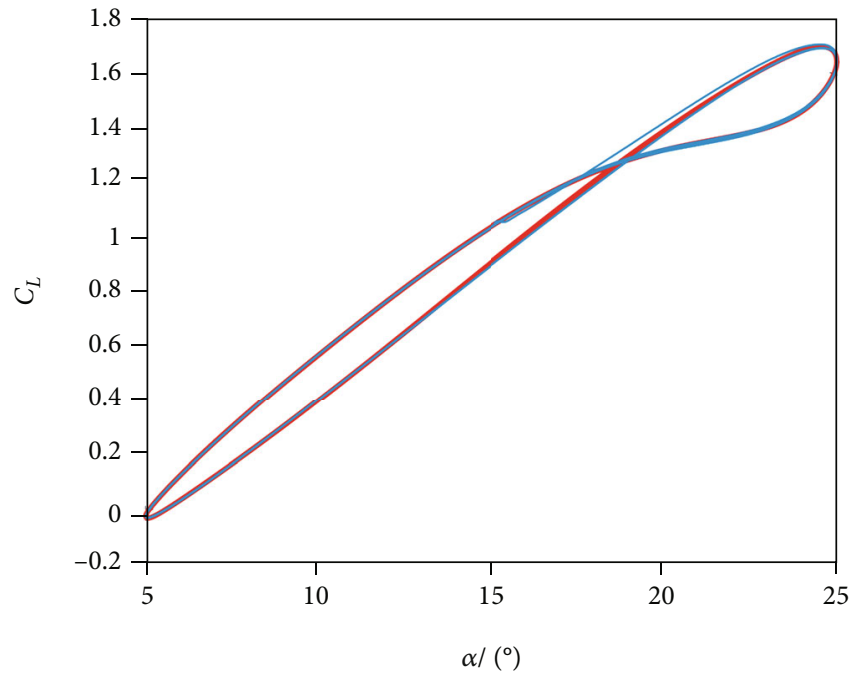

(a) Lift coefficient

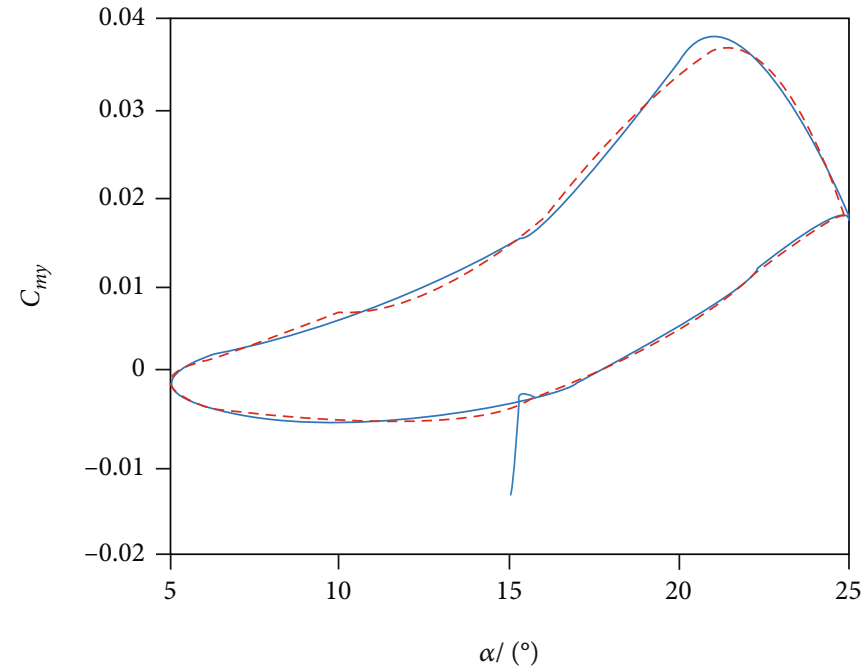

- - Model data

- CFD result

FIgURE 9: Predictions of the fourth-order model $(k=0.04)$.

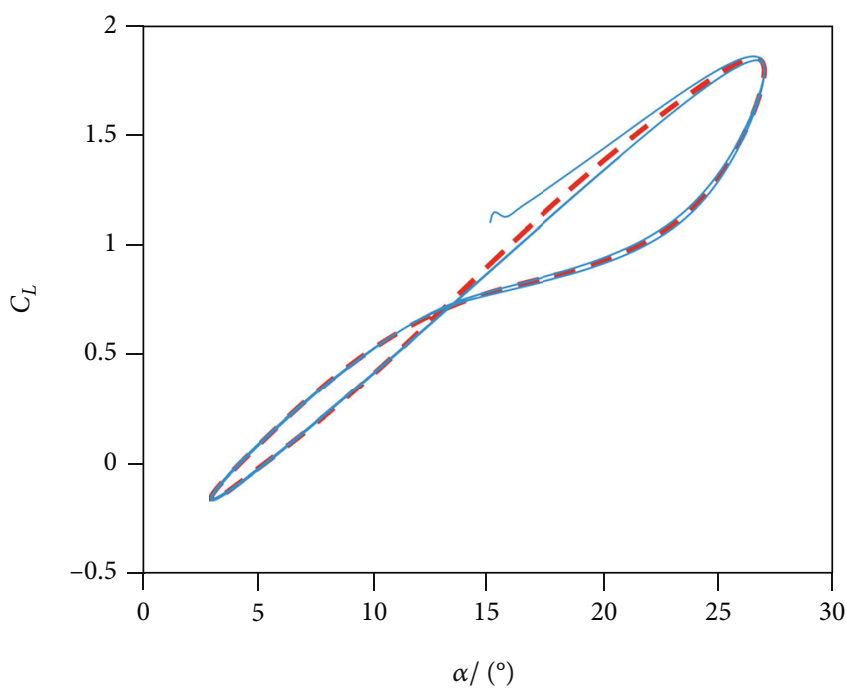

(a) Lift coefficient

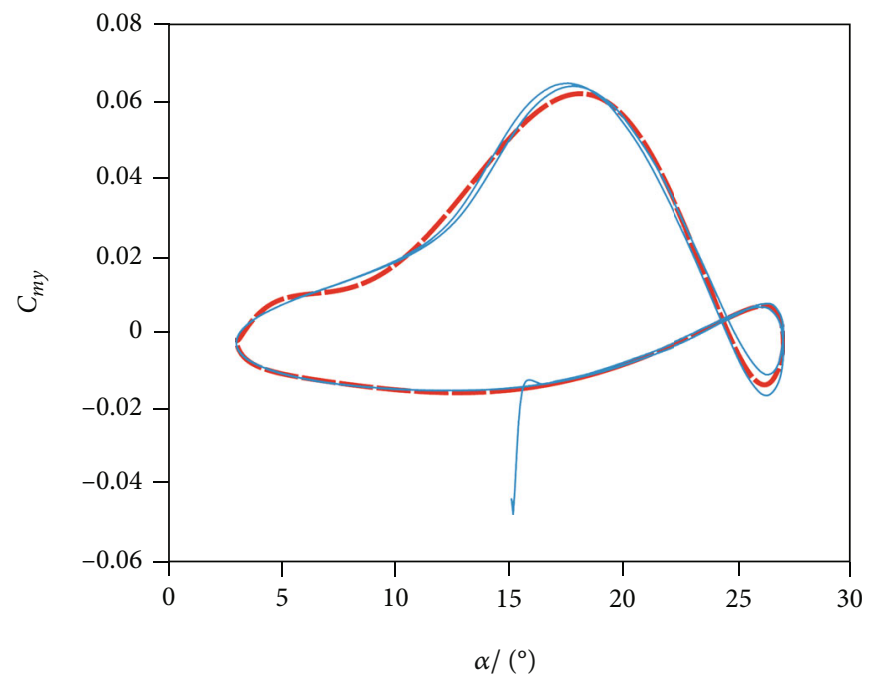

- - - Model data

CFD result

Figure 10: Predictions of the fourth-order model $\alpha_{m}=12^{\circ}$. 


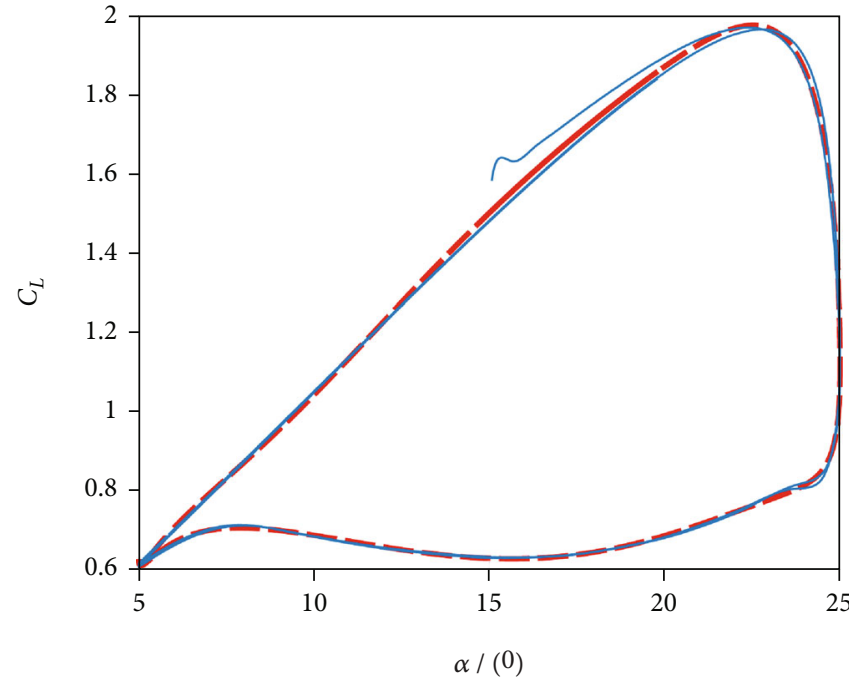

- - - Model data

- CFD result

(a) Lift coefficient

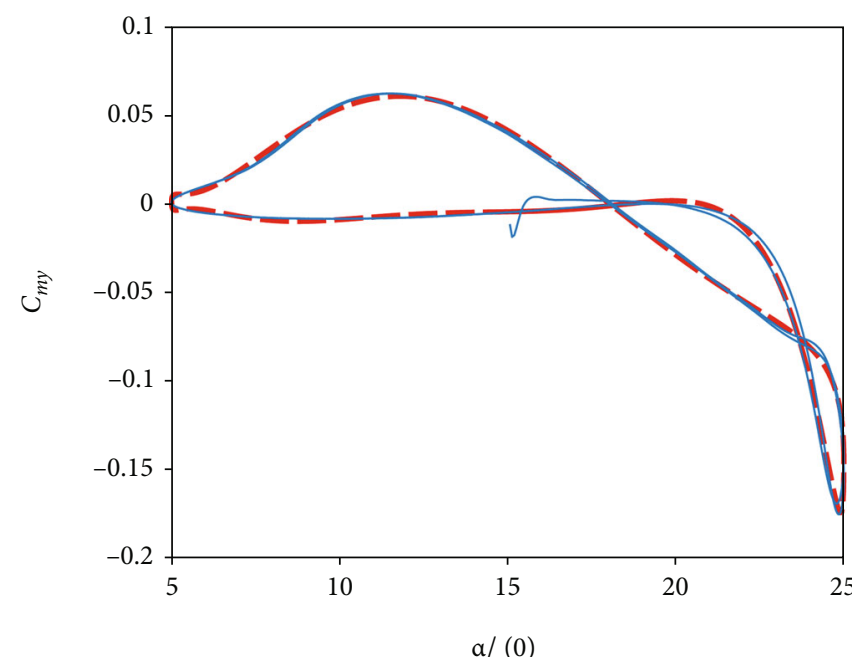

- - - Model data CFD result

(b) Pitch moment coefficient

Figure 11: Predictions of the fourth-order model $\alpha_{0}=12^{\circ}$.

$$
\begin{aligned}
C_{i}= & C_{0}+C_{1}\left(\alpha_{0}, k, \alpha_{m}\right) \alpha+C_{2}\left(\alpha_{0}, k, \alpha_{m}\right) \alpha^{2}+C_{3}\left(\alpha_{0}, k, \alpha_{m}\right) \dot{\alpha} \\
& +C_{4}\left(\alpha_{0}, k, \alpha_{m}\right)|\dot{\alpha}|+C_{5}\left(\alpha_{0}, k, \alpha_{m}\right) \dot{\alpha} \dot{\alpha} \\
& +C_{6}\left(\alpha_{0}, k, \alpha_{m}\right) \alpha|\dot{\alpha}|+C_{7}\left(\alpha_{0}, k, \alpha_{m}\right)|\dot{\alpha}| \dot{\alpha} \\
& +C_{8}\left(\alpha_{0}, k, \alpha_{m}\right) \dot{\alpha}^{2}+C_{9}\left(\alpha_{0}, k, \alpha_{m}\right) \alpha^{3} \\
& +C_{10}\left(\alpha_{0}, k, \alpha_{m}\right) \dot{\alpha} \alpha^{2}+C_{11}\left(\alpha_{0}, k, \alpha_{m}\right)|\dot{\alpha}| \alpha^{2} \\
& +C_{12}\left(\alpha_{0}, k, \alpha_{m}\right) \dot{\alpha}^{2} \alpha+C_{13}\left(\alpha_{0}, k, \alpha_{m}\right)|\dot{\alpha}| \dot{\alpha} \alpha \\
& +C_{14}\left(\alpha_{0}, k, \alpha_{m}\right)|\dot{\alpha}| \dot{\alpha}^{2}+C_{15}\left(\alpha_{0}, k, \alpha_{m}\right) \dot{\alpha}^{3} .
\end{aligned}
$$

(3) The fourth-order model:

$$
\begin{aligned}
C_{i}= & C_{0}+C_{1}\left(\alpha_{0}, k, \alpha_{m}\right) \alpha+C_{2}\left(\alpha_{0}, k, \alpha_{m}\right) \alpha^{2}+C_{3}\left(\alpha_{0}, k, \alpha_{m}\right) \dot{\alpha} \\
& +C_{4}\left(\alpha_{0}, k, \alpha_{m}\right)|\dot{\alpha}|+C_{5}\left(\alpha_{0}, k, \alpha_{m}\right) \dot{\alpha} \dot{\alpha}+C_{6}\left(\alpha_{0}, k, \alpha_{m}\right) \alpha|\dot{\alpha}| \\
& +C_{7}\left(\alpha_{0}, k, \alpha_{m}\right)|\dot{\alpha}| \dot{\alpha}+C_{8}\left(\alpha_{0}, k, \alpha_{m}\right) \dot{\alpha}^{2}+C_{9}\left(\alpha_{0}, k, \alpha_{m}\right) \alpha^{3} \\
& +C_{10}\left(\alpha_{0}, k, \alpha_{m}\right) \dot{\alpha} \alpha^{2}+C_{11}\left(\alpha_{0}, k, \alpha_{m}\right)|\dot{\alpha}| \alpha^{2} \\
& +C_{12}\left(\alpha_{0}, k, \alpha_{m}\right) \dot{\alpha}^{2} \alpha+C_{13}\left(\alpha_{0}, k, \alpha_{m}\right)|\dot{\alpha}| \dot{\alpha} \alpha \\
& +C_{14}\left(\alpha_{0}, k, \alpha_{m}\right)|\dot{\alpha}| \dot{\alpha}^{2}+C_{15}\left(\alpha_{0}, k, \alpha_{m}\right) \dot{\alpha}^{3} \\
& +C_{16}\left(\alpha_{0}, k, \alpha_{m}\right) \alpha^{4}+C_{17}\left(\alpha_{0}, k, \alpha_{m}\right) \dot{\alpha} \alpha^{3} \\
& +C_{18}\left(\alpha_{0}, k, \alpha_{m}\right)|\dot{\alpha}| \alpha^{3}+C_{19}\left(\alpha_{0}, k, \alpha_{m}\right)|\dot{\alpha}| \dot{\alpha} \alpha^{2} \\
& +C_{20}\left(\alpha_{0}, k, \alpha_{m}\right) \dot{\alpha}^{2} \alpha^{2}+C_{21}\left(\alpha_{0}, k, \alpha_{m}\right) \dot{\alpha}^{3} \alpha \\
& +C_{22}\left(\alpha_{0}, k, \alpha_{m}\right)|\dot{\alpha}|^{3} \alpha+C_{23}\left(\alpha_{0}, k, \alpha_{m}\right)|\dot{\alpha}| \dot{\alpha}^{3} \\
& +C_{24}\left(\alpha_{0}, k, \alpha_{m}\right) \dot{\alpha}^{4} .
\end{aligned}
$$

With the introduction of the new functional relationship, the aerodynamic coefficient becomes a function related

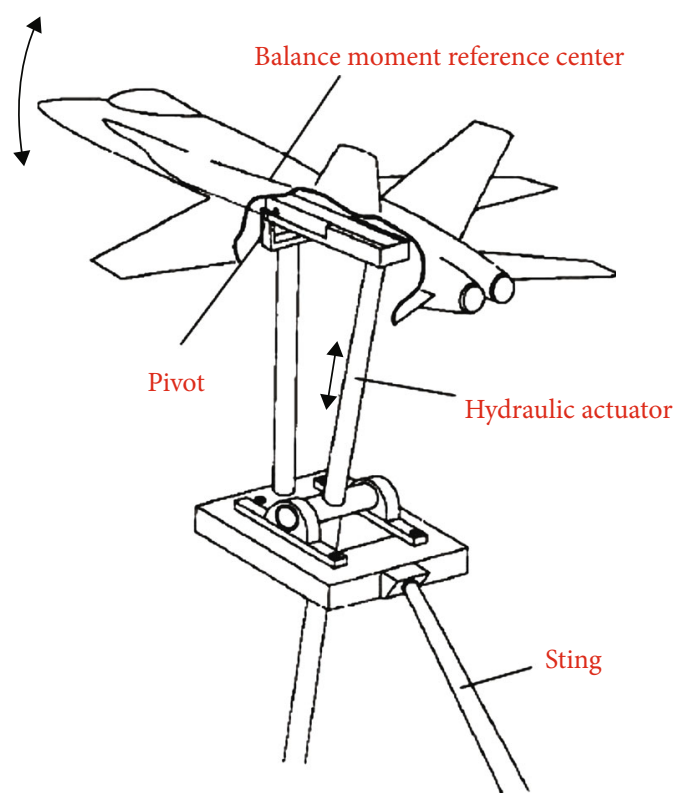

Figure 12: F-18 model.

to the aerodynamic parameters, which can represent the possible nonlinear relationship to a certain extent. Because there are many undetermined parameters, the optimization algorithm can be used to identify them.

4.3. Parameter Identification. The undetermined parameters in the model are $a_{i 0}, a_{i 1}, a_{i 2}, a_{i 3}, \cdots$ Various optimization algorithms can be used for parameter identification. This paper uses the particle swarm optimization (PSO) algorithm [23] to optimize the cumulative error between the predicted value and reference value of the target bit model. 


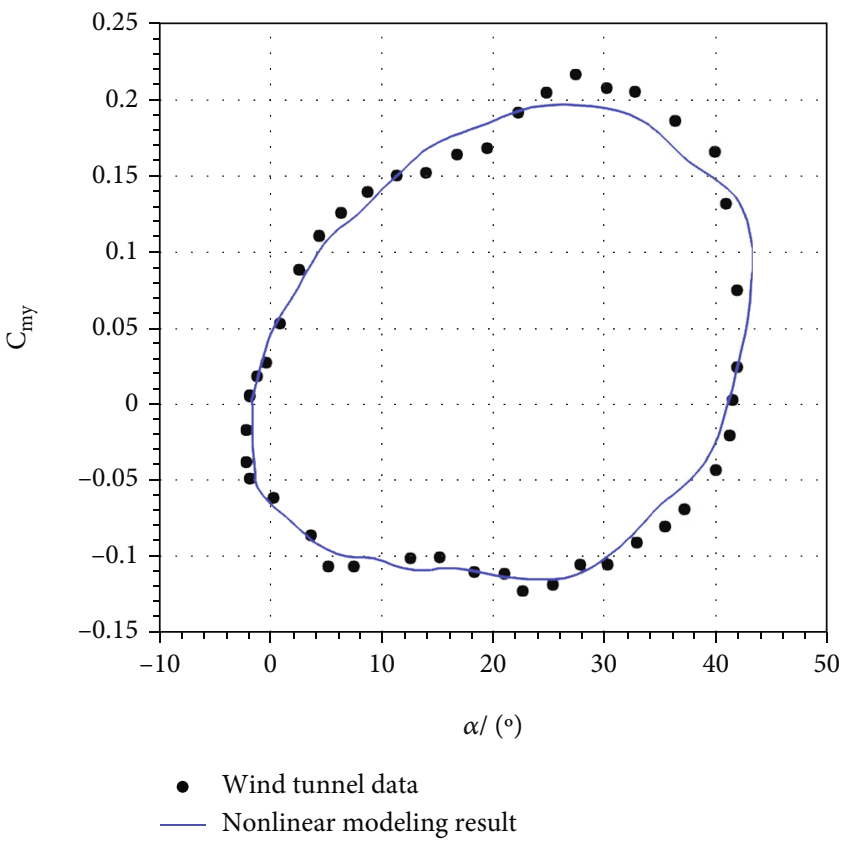

FIgURE 13: Comparison between the predicted results and wind tunnel data.

Note that the time range of unsteady motion is $0<t<T$. The corresponding instantaneous angle of attack and its rate of change are $\alpha(t), \dot{\alpha}(t)$. CFD calculations are $\widehat{C}_{i}\left(t_{j}\right)(j=1$, $2, \cdots, n)$. The predicted value of the model is $C_{i}\left(t_{j}\right)(j=1,2$, $\cdots, n)$. Then, the overall prediction error is

$$
\varepsilon=\sum_{j=1}^{l}\left[C_{i}\left(t_{j}\right)-C \wedge_{i}\left(t_{j}\right)\right]^{2}
$$

\section{Validation Analysis of Improved Model}

5.1. Validation of Models with Different Expansion Orders. The large amplitude oscillation of the NACA 0015 airfoil is used to test the prediction ability of the above models. The calculation conditions are the same as those above. The initial angle of attack is $9^{\circ}$, and the amplitude is $10^{\circ}$. First, the modified models with different expansion times are verified. The reduced frequency is $k=0.06$, and the comparison between the unsteady aerodynamic forces predicted by the second-, third-, and fourth-order models and the CFD calculations is shown in Figures 6-8. It can be seen that with the increase of the expansion times, the prediction accuracy of the improved model also improved, but the cost of parameter identification is also greater, so the expansion times should not be too high. According to the results of this paper, the prediction accuracy of the fourth-order model basically meets the requirements.

5.2. Aerodynamic Modeling at Different Reduced Frequencies. The improved model takes into account the influence of the reduced frequency on the aerodynamic derivative, so the unsteady aerodynamic forces at other reduced frequencies can be predicted according to the existing reduced frequency aerodynamic data. The unsteady aerodynamic force at a reduced frequency of $k=0.04$ is predicted by using the fourth-order model based on the aerodynamic data of $k=$ $0.02,0.06,0.08$. The predictions of the lift and pitch moment coefficients are shown in Figure 9. The results show that the predicted values are in good agreement with the CFD calculations, which proves the prediction ability of the new model for aerodynamic forces at different reduction frequencies. Because the flow field characteristics of the selected reduced frequency are similar, it is better to predict the unsteady aerodynamic force at another reduced frequency through several reduced frequencies. Note also that the more sample points sampled, the more accurate the model is, and the more accurate the prediction of the unsteady aerodynamic force is.

5.3. Aerodynamic Modeling at Different Amplitudes. The improved model is used to predict unsteady aerodynamic forces at different amplitudes. The unsteady aerodynamic force at an amplitude of $\alpha_{m}=12^{\circ}$ is predicted by using the aerodynamic data of $\alpha_{m}=10^{\circ}, \alpha_{m}=14^{\circ}$, and $\alpha_{m}=15^{\circ}$. Figure 10 shows that the nonlinear characteristics of the aerodynamic force are more clear when the amplitude is $12^{\circ}$. At this time, the prediction accuracy of the fourth model is relatively high, but the prediction effect in local areas still needs to be improved.

5.4. Aerodynamic Modeling at Different Initial Angles of Attack. The improved model is used to predict the unsteady aerodynamic forces at different initial angles of attack. The lift and pitch moment coefficients of the unsteady pitching vibration at the initial angle of attack of $\alpha_{0}=12^{\circ}$ are predicted by using the aerodynamic data of $\alpha_{0}=9^{\circ}, \alpha_{0}=15^{\circ}$, and $\alpha_{0}=18^{\circ}$. The results in Figure 11 show that the nonlinearity of the unsteady aerodynamic force is significantly enhanced at an initial angle of attack of $\alpha_{0}=12^{\circ}$. and the 
prediction accuracy of the quad model is still high. In addition, the predicted flow field characteristics are within the range of the modeled sample data, so the improved model can obtain relatively ideal results. That is, from the perspective of "interpolation" prediction, the improved model has a better effect.

Compared with the original model, this model takes into account more flow field influence parameters. Although the model is modified in the form of simple harmonic vibration, the general motion can also be transformed into the equivalent harmonic form. Therefore, the parameters of the improved nonlinear model are generalized.

5.5. Aerodynamic Modeling Validation of the F-18 Model. To further validate the new nonlinear aerodynamic model, the wind tunnel results of the F-18 model (Figure 12) were used to construct the numerical test. Dynamic data at a high angle of attack are obtained from the NASA Langley Research Center [1]. All the experiments are proposed with a flow speed of $V=72 \mathrm{~m} / \mathrm{s}$, and the relative Reynolds number is $\operatorname{Re}=0.4 \times 10^{6}$. The dynamic cases of different reduced frequencies $(k=0.0558, k=0.0782$, and $k=0.0116)$ are used to identify the nonlinear model and further predict the unsteady aerodynamic moments in the case of $k=0.0892$. Figure 13 shows that the predicted results are uniform with the wind tunnel data, which indicates that the new model is effective and can be applied to various configurations.

\section{Conclusions}

To improve the nonlinear aerodynamic modeling ability at a high angle of attack, this study developed an extended nonlinear aerodynamic derivative model by expanding the higher-order terms and adding three more motion parameters to correct the constant expression of the coefficient terms in the classical model. Some conclusions can be drawn:

(1) When the dynamic flow field is linear or weakly nonlinear, the original aerodynamic derivative model can accurately predict the unsteady aerodynamic force, and the assumption of a constant aerodynamic derivative is applicable

(2) When the flow field is highly nonlinear, the simple original model cannot describe this change, and the predicted aerodynamic force is completely inconsistent with the reference value

(3) When the higher-order terms are expanded and the aerodynamic derivative is nonlinearly modified, the prediction ability of the newly established aerodynamic derivative model at high angles of attack is greatly improved. Additionally, the higher the expansion order is, the more accurate the prediction of the aerodynamic forces at high angles of attack. At the same time, the new model can predict the unsteady aerodynamic forces under other operating conditions using the existing aerodynamic data of the initial angle of attack, reduced frequency, or oscillation amplitude

In conclusion, the aerodynamic derivative model can still play a role in the analysis of the unsteady dynamic aerodynamic characteristics at high angles of attack after modification and improvement. Moreover, if more nonlinear influencing factors of high angles of attack can be extracted and the aerodynamic derivative model can be improved, the application scope and prediction ability of the model at high angles of attack will be further improved. Note that the model can also be extended to the aerodynamic prediction in the lateral direction.

\section{Data Availability}

Data available are on request

\section{Conflicts of Interest}

The authors declare that they have no financial and personal relationships with other people or organizations that can inappropriately influence our work and that there is no professional or other personal interest of any nature or kind in any products, service, and/or company that could be construed as influencing the position presented in, or the review of, the manuscript entitled.

\section{Acknowledgments}

The authors would like to acknowledge the support of the Fundamental Research Funds for the Central Universities (Grant No. G2020KY05115) and the support of Natural Science Basic Research Program of Shaanxi (Program No. 2021JQ-084).

\section{References}

[1] B. G. Mi, H. Zhan, and S. S. Lu, "An extended unsteady aerodynamic model at high angles of attack," Aerospace Science and Technology, vol. 77, pp. 788-801, 2018.

[2] M. R. Visbal and J. S. Shang, "Investigation of the flow structure around a rapidly pitching airfoil," AIAA Journal, vol. 27, no. 8, pp. 1044-1051, 2012.

[3] Y. M. Rizk and K. Gee, "Unsteady simulation of viscous flowfield around F-18 aircraft at large incidence," Journal of Aircraft, vol. 29, no. 6, pp. 986-992, 1992.

[4] A. A. Elmiligui, K. S. Abdol-Hamid, and E. B. Parlette, "Detached eddy simulation for the F-16XL aircraft configuration," in 53rd Aerospace Sciences Meeting, Kissimmee, Florida, 2015.

[5] O. J. Boelens, "CFD analysis of the flow around the X-31 aircraft at high angle of attack," Aerospace Science and Technology, vol. 20, no. 1, pp. 38-51, 2012.

[6] S. H. Lee and Y. O. Han, "Experimental investigation of highangle-of-attack aerodynamics of low-aspect-ratio rectangular wings configured with NACA0012 airfoil section," International Journal of Aeronautical and Space Sciences, vol. 21, no. 2, pp. 303-314, 2020.

[7] A. Tewari, Unsteady Aerodynamic Modeling, Springer, New York, 2015. 
[8] M. Ghoreyshi, A. Jirasek, and R. M. Cummings, "Reduced order unsteady aerodynamic modeling for stability and control analysis using computational fluid dynamics," Progress in Aerospace Sciences, vol. 71, pp. 167-217, 2014.

[9] B. G. Mi, H. Zhan, and B. B. Chen, "New systematic CFD methods to calculate static and single dynamic stability derivatives of aircraft," Mathematical Problems in Engineering, vol. 2017, 11 pages, 2017.

[10] G.-F. Lin, C. Lan, J. Brandon, G.-F. Lin, C. Lan, and J. Brandon, "A generalized dynamic aerodynamic coefficient model for flight dynamics applications," in 22nd Atmospheric Flight Mechanics Conference, New Orleans, LA, 1997.

[11] M. Goman and A. Khrabrov, "State-space representation of aerodynamic characteristics of an aircraft at high angles of attack," Journal of Aircraft, vol. 31, no. 5, pp. 1109-1115, 2012.

[12] N. Abramov, A. Khrabrov, K. Kolinko, and M. Goman, "Simple wings unsteady aerodynamics at high angles of attack experimental and modeling results," in 24th Atmospheric Flight Mechanics Conference, Portland, OR, 1999.

[13] N. Abramov, M. Goman, M. Demenkov, and A. Khrabrov, "Aircraft dynamics at high incidence flight with account of unsteady aerodynamic effects," in AIAA Atmospheric Flight Mechanics Conference \& Exhibit, Providence, Rhode Island, 2004.

[14] X. Huang and E. Hanff, "Non-linear indicial response and internal state-space representation for free-to-roll trajectory prediction of a 65 degree wing at high incidence," in AIAA Atmospheric Flight Mechanics Conference \& Exhibit, Monterey, California, 2013.

[15] M. Ghoreyshi, R. M. Cummings, A. D. Ronch, and K. J. Badcock, "Transonic aerodynamic load modeling of X-31 aircraft pitching motions," AIAA Journal, vol. 51, no. 10, pp. 24472464, 2013.

[16] T. Farsadi, M. Rahmanian, and A. Kayan, "Reduced order nonlinear aeroelasticity of swept composite wings using compressible indicial unsteady aerodynamics," Journal of Fluids and Structures, vol. 92, p. 102812, 2020.

[17] S. CHEN, Z. GAO, X. ZHU, Y. du, and C. PANG, "Unstable unsteady aerodynamic modeling based on least squares support vector machines with general excitation," Chinese Journal of Aeronautics, vol. 33, no. 10, pp. 2499-2509, 2020.

[18] Y. Jiang, Q. Zhao, and J. Zhu, "Unsteady aerodynamics modeling using SVM and artificial neural network," Lecture Notes in Electrical Engineering, vol. 336, pp. 577-585, 2015.

[19] S. Ananthan, G. Vijayakumar, and S. Yellapantula, “A DNN surrogate unsteady aerodynamic model for wind turbine loads calculations," Journal of Physics Conference Series, vol. 1618, article 052060, 2020.

[20] M. Winter and C. Breitsamter, "Nonlinear identification via connected neural networks for unsteady aerodynamic analysis," Aerospace science and technology, vol. 77, pp. 802-818, 2018.

[21] K. Li, J. Kou, and W. Zhang, "Deep neural network for unsteady aerodynamic and aeroelastic modeling across multiple Mach numbers," Nonlinear Dynamics, vol. 96, no. 3, pp. 2157-2177, 2019.

[22] A. Qasrawi and R. A. East, "Measurements of dynamic stability derivatives of hyperballistic and conic shapes at $\mathrm{M}=6.85$," Aeronautical Quarterly, vol. 32, no. 1, pp. 1-30, 2016.

[23] B. G. Mi and X. Y. Wang, "A new aerodynamic optimization method with the consideration of dynamic stability," International Journal of Aerospace Engineering, vol. 2021, 9 pages, 2021. 